\title{
Retos en el abordaje de la vía biliar en épocas de COVID-19 del Servicio de Endoscopia Gastrointestinal del Hospital General de México
}

\author{
Juan C. Silis-Cravioto*, Miguel Á. Herrera-Servín, Edwin Ornelas-Escobedo, Elymir S. Galvis-García, \\ Viridiana Oregel-Aguilar y Julio C. Zavala-Castillo \\ Servicio de Endoscopia Gastrointestinal, Hospital General de México Dr. Eduardo Liceaga, Ciudad de México, México
}

\begin{abstract}
Resumen
Introducción: Los servicios de endoscopia de los diferentes hospitales en nuestro país se han visto afectados debido a la contingencia, encontrándose detenidos por la sobrecarga de atención de pacientes con enfermedad por coronavirus 2019 (COVID-19), dejando a un lado el manejo endoscópico. Objetivo: Evaluar la factibilidad de implementación de los protocolos de seguridad biológica y su eficacia para prevenir infecciones asociadas al coronavirus 2, causante del síndrome respiratorio agudo grave (SARS-CoV-2). Material y métodos: Se observaron los procedimientos de CPRE durante la contingencia COVID-19 entre el 23 de marzo hasta el 15 de junio del 2020. Resultados: Se realizaron 36 procedimientos de CPRE siendo el diagnóstico más frecuente fue la dilatación de la vía biliar (58.3\%). El principal procedimiento realizado fue la esfinterotomía (61.1\%) adoptándose el 100\% de las medidas de seguridad. Conclusiones: La realización de la CPRE es factible y segura durante la época de COVID-19, sin afectar las tasas de éxito endoscópico, logrando resultados satisfactorios con riesgo nulo de contagio para el personal de la salud.
\end{abstract}

Palabras clave: CPRE. Contingencia. COVID-19. Equipos de protección personal.

\section{Challenges in approaching the bile duct in times of COVID-19 for the gastrointestinal endoscopy department at the General Hospital of Mexico}

\begin{abstract}
Introduction: The endoscopy services of the different hospitals in our country have been affected due to the contingency, being detained due to the overload of care of patients with coronavirus disease 2019 (COVID-19), leaving aside endoscopic management. Objective: To evaluate the feasibility of implementing biological safety protocols and their efficacy to prevent infections associated with coronavirus 2, which causes severe acute respiratory syndrome (SARS-CoV-2). Material and methods: ERCP procedures were observed during the COVID-19 contingency, between March 23 to June 15, 2020. Results: 36 ERCP procedures were performed, the most frequent diagnosis being bile duct dilation (58.3\%). The main procedure performed was sphincterotomy $(61.1 \%)$, adopting $100 \%$ of the safety measures. Conclusions: The performance of ERCP is feasible and safe during the COVID-19 era, without affecting endoscopic success rates, achieving satisfactory results with zero risk of contagion for health personnel.
\end{abstract}

Key words: ERCP. Contingency. COVID-19. Personal protective equipment.

Correspondencia:

*Juan C. Silis-Cravioto

E-mail: juan.silis@ hotmail.com
Fecha de recepción: 21-07-2020

Fecha de aceptación: 20-09-2020 DOI: 10.24875/END.20000059
Disponible en internet: 06-11-2020 Endoscopia. 2020;32(4):120-124 www.endoscopia-ameg.com 


\section{Introducción}

La enfermedad por coronavirus 2019 (COVID-19) es potencialmente letal y por su rápido esparcimiento y compromiso mundial, la Organización Mundial de la Salud la ha declarado pandemia ${ }^{1}$. A pesar de que el personal de atención médica que trabaja en las unidades de endoscopia no está directamente involucrado en la evaluación diagnóstica y terapéutica de los pacientes positivos para el coronavirus 2 del síndrome respiratorio agudo grave (SARS-CoV-2), la endoscopia debe considerarse como un procedimiento riesgoso, debido a la emisión de aerosoles (eructos, vómitos, flatos y presencia de materia fecal), aunque no se dispone de datos definitivos sobre si la insuflación positiva durante los procedimientos endoscópicos y la manipulación de los accesorios pueden aumentar el riesgo de aerosolización de fluidos biológicos de los pacientes. Es por esta razón que la transmisión del SARS-CoV-2 durante la colangiopancreatografía retrógrada endoscópica (CPRE) podría ser particularmente alta. De hecho, los pacientes a menudo requieren intubación orotraqueal, con alto riesgo de generar aerosoles. Los procedimientos son frecuentemente complejos y pueden requerir una corta distancia física entre endoscopista y paciente durante un periodo de tiempo que generalmente es más largo que en la gastroscopia y colonoscopia estándar; el uso de varios accesorios y la necesidad de intercambio frecuente de dispositivos, como pinzas, trampas, redes, cables guía y globos puede aumentar el riesgo de esparcir fluidos biológicos y aumentar la tasa de exposición ${ }^{2}$.

La CPRE requiere de la participación al mismo tiempo de varios integrantes, aumentando el riesgo de contaminación concomitante. El complejo diseño de los duodenoscopios dificulta la limpieza y posibilita la formación de biopelículas que promueven el crecimiento excesivo de microorganismos ${ }^{3}$.

De acuerdo con las sociedades endoscópicas internacionales, la CPRE no debe posponerse en condiciones potencialmente mortales. En el caso de colangitis, obstrucción pancreatobiliar, fugas biliares 0 pancreáticas postraumáticas o postoperatorias, colecistitis no elegible para cirugía y acumulaciones de líquido pancreático sintomáticas o infectadas, los riesgos de morbilidad y mortalidad son altos, por lo que la endoscopia debe realizarse ${ }^{4}$.

Para reducir el riesgo de transmisión del SARS-CoV2 se ha demostrado que la higiene adecuada de manos y el uso de equipos de protección personal (EPP) es eficaz para reducir el riesgo de transmisión. El objetivo de los
EPP es crear una barrera entre el material potencialmente peligroso y el trabajador sanitario, siendo el uso obligatorio para todo el personal de la unidad de endoscopia. El EPP avanzado incluye mascarilla, doble guante, protección para los ojos (gafas protectoras o careta), cubierta para el cabello, cubrezapatos y bata de aislamiento 4 .

En el servicio de endoscopia de este hospital, a pesar de la contingencia y el riesgo alto de contagio, se continúa realizando estudios endoscópicos de urgencia a los pacientes con patología biliar, siendo el objetivo del presente trabajo evaluar la factibilidad de implementación de los protocolos de seguridad biológica y su eficacia para prevenir infecciones asociadas al SARS-CoV-2, además de los retos al utilizar estas medidas y sus complicaciones, mencionando las indicaciones de CPRE, sus hallazgos y técnicas terapéuticas realizadas durante la contingencia.

\section{Material y métodos}

Se realizó un estudio en el Hospital General de México Dr. Eduardo Liceaga, donde se observaron los procedimientos de CPRE durante la contingencia COVID-19 entre el 23 de marzo hasta el 15 de junio del 2020. Se realizó un estudio retrospectivo, longitudinal y descriptivo en pacientes de cualquier edad, de ambos sexos, sin importar el estatus de COVID-19, que contasen con indicación para CPRE durante el periodo de contingencia.

Se registró la información de los pacientes, de acuerdo con las características demográficas, estado de salud a su ingreso y egreso del servicio, incluyendo su indicación, tratamiento y complicaciones durante el estudio, además de las medidas de protección e insumos utilizados.

En todos los pacientes se utilizó mascarilla quirúrgica, la cual se retiró justo antes de comenzar el procedimiento. Por parte del personal se utilizaron las medidas y los EPP proporcionados por nuestra unidad hospitalaria durante cada uno de los estudios de endoscopia, como el uso de gorro quirúrgico, ropa quirúrgica (pijama y bata quirúrgica), cubrebocas o mascarilla facial N95, protección ocular con gafas o goggles, así como el uso de careta, además de doble guante que abarque muñeca y bata, todos ellos siguiendo la normativa de colocación y retiro previo lavado de manos. De las cinco salas que se cuentan en el servicio, solo una de ellas se utilizó para CPRE, así como para pacientes sospechosos de COVID-19, separando esa área del resto para evitar una contaminación cruzada. De igual manera, se redujo el número de personas 
durante el estudio, contando solo con el endoscopista encargado, residente, enfermera, anestesiólogo y técnico de rayos $X$. Al endoscopio se le realizó limpieza por el médico endoscopista al término del estudio, además del reprocesamiento de endoscopios y accesorios mediante equipos de reprocesamiento. La sala endoscópica fue descontaminada mediante la limpieza de la habitación con soluciones alcoholadas al $70 \%$. Una vez terminado el proceso de limpieza se utilizó luz ultravioleta avanzada (UV-360) durante un periodo de $10 \mathrm{mi}-$ nutos para garantizar un ambiente libre de virus, todo esto basado en las recomendaciones para las unidades de endoscopia de la Sociedad Interamericana de Endoscopia Digestiva.

Se utilizó estadística descriptiva con cálculo de medidas de tendencia central (media y mediana) y de dispersión (desviación estándar y rango intercuartil), además de medidas de proporción. Los datos fueron analizados utilizando el programa SPSS Statistics v.17.0.

\section{Resultados}

Se realizaron en el periodo de estudio 36 procedimientos de CPRE, donde se observó un predominio de mujeres $(52.7 \%$ ) en este procedimiento. La mediana de edad fue de 49 años (desviación estándar [DE]: \pm 20.2; rango intercuartílico: 36.25).

Los diagnósticos más frecuentes obtenidos tras la realización de CPRE fueron: dilatación de la vía biliar $(58.3 \%)$, seguida de coledocolitiasis $(47.2 \%)$ y colangitis $(19.4 \%)$. Las estenosis benignas (11.1\%), la lesión benigna de la vía biliar (8.8\%), el cáncer de páncreas (5.5\%) y la hipertrofia del esfínter de Oddi $(2.7 \%)$ fueron otros de los hallazgos encontrados. El $13.8 \%$ de los procedimientos resultaron no satisfactorios 0 fallidos debido a edema de papila al momento de la canulación de la vía biliar principal. En las CPRE terapéuticas encontramos que el principal procedimiento fue la esfinterotomía $(61.1 \%)$, seguida por la colocación de prótesis biliares $(58.3 \%)$. Entre otros procedimientos realizados encontramos el barrido con balón para la extracción de litos y el precorte en un $25 \%$.

Del total de pacientes con coledocolitiasis el porcentaje resuelto por CPRE fue el $81.25 \%$; el $18.75 \%$ restante presentó desproporción lito-colédoco, resuelta parcialmente mediante colocación de prótesis biliar (Tabla 1). El $69.4 \%$ fueron considerados como técnicas de canulación difícil (Tabla 2). La mediana del tiempo de estudio fue de 25 minutos (DE: \pm 9.3 ). Aquellos que presentaron alguna disfunción orgánica o sistémica al momento del estudio correspondieron al $41.6 \%$. Entre las complicaciones se presentaron $4(11.1 \%)$ casos de sangrado posterior a la esfinterotomía, las cuales se resolvieron con inyección de sustancias vasoconstrictoras. La pancreatitis post-CPRE se presentó en el $5.5 \%$ de los casos; cabe mencionar que en todos los pacientes se utilizó indometacina rectal $100 \mathrm{mg}$ previo al estudio. Se encontró 1 complicación grave (2.7\%): una perforación suprapapilar que fue resuelta mediante la utilización de clip metálico. La mortalidad fue de un paciente con CPRE resolutiva, debido a sangrado en capa en múltiples sitios del tracto digestivo superior, el cual falleció aproximadamente una hora después del estudio.

Las medidas de seguridad (dentro de las que abarcan el uso de gorro quirúrgico, gafas o goggles, careta, cubrebocas, guantes y ropa quirúrgica [pijama y bata quirúrgica]) fueron adoptadas por el $100 \%$ de los miembros del equipo. En cuanto a la desinfección de los equipos y la descontaminación de la sala de endoscopia, encontramos que igualmente el $100 \%$ se realizó acorde a las guías internacionales previamente estandarizadas.

El reto que presentó cada uno de los médicos al momento de desarrollar el procedimiento de CPRE fue la poca visualización, ya que los dispositivos de protección ocular se empañaban a medida que se utilizaban y se desarrollaba el procedimiento, la falta de aire o disnea secundario al uso de cubrebocas herméticos y caretas, además de la poca movilidad debido al equipo de protección; sin embargo, estas eventualidades no incrementaron la complejidad de la CPRE ni el número de complicaciones.

Del total del personal (médicos adscritos, residentes y enfermería) desde el inicio de la contingencia en nuestro país y desde la transformación del hospital en receptor COVID, el servicio de endoscopia reportó un $0 \%$ de contagios mediante un seguimiento de síntomas que previamente el personal médico llevaba de manera individual, evaluándolo a cada uno de ellos hasta el final del estudio, reportando que todo el personal permaneció asintomático. Las pruebas de reacción en cadena de la polimerasa con transcriptasa inversa (PCR-RT) solo se realizaron en aquellos que estuvieron expuestos a pacientes con COVID-19, de los cuales ninguno ameritó dicha prueba.

\section{Discusión}

De acuerdo con los resultados obtenidos en el estudio, en cuanto a datos demográficos se evidenció que la población de pacientes en la que se realiza un mayor número de CPRE es mayoritariamente del sexo femenino, sobre todo en la $5 .^{a}$ década de la vida. Estos 
Tabla 1. Resultados del manejo de la coledocolitiasis por colangiopancreatografía retrógrada endoscópica en el Hospital General de México Dr. Eduardo Liceaga

\begin{tabular}{|l|c|c|}
\multicolumn{3}{|c|}{$\begin{array}{c}\text { Resultados del manejo de la coledocolitiasis por } \\
\text { colangiopancreatografía retrógrada endoscópica en el } \\
\text { Hospital General de México Dr. Eduardo Liceaga }\end{array}$} \\
\hline Manejo de coledocolitiasis & Frecuencia & Porcentaje \\
\hline Resuelta & 13 & $81.25 \%$ \\
\hline Parcialmente resuelta & 3 & $18.75 \%$ \\
\hline Total & 16 & $100 \%$ \\
\hline
\end{tabular}

Tabla 2. Canulación difícil en pacientes sometidos a colangiopancreatografía retrógrada endoscópica en el Hospital General de México Dr. Eduardo Liceaga

\begin{tabular}{|l|c|c|}
\hline \multicolumn{3}{|c|}{$\begin{array}{c}\text { Canulación difícil en pacientes sometidos a } \\
\text { colangiopancreatografía retrógrada endoscópica en el } \\
\text { Hospital General de México Dr. Eduardo Liceaga }\end{array}$} \\
\hline Técnica de canulación & Frecuencia & Porcentaje \\
\hline Canulación difícil & 25 & $69.4 \%$ \\
\hline Canulación selectiva & 11 & $30.6 \%$ \\
\hline Total & 36 & $100 \%$ \\
\hline
\end{tabular}

hallazgos son concordantes con la literatura publicada, en la cual se aprecia una mayor prevalencia de la enfermedad a una mayor edad de los pacientes llevados a CPRE $^{5}$.

Las indicaciones más comunes para la realización de CPRE fueron la sospecha de coledocolitiasis y colangitis, encontrando como hallazgo principal la dilatación de la vía biliar. Al seleccionar a los pacientes con litiasis y dilatación biliar encontramos que de acuerdo con las guías de la American Society for Gastrointestinal Endoscopy 6 el riesgo de coledocolitiasis se incrementa hasta en un $89 \%$ de los pacientes con hallazgos de dilatación por ultrasonido previo al estudio de CPRE. En nuestro estudio el porcentaje que se presentó lito en una vía biliar dilatada fue similar a los datos obtenidos en estudios similares ${ }^{7}$.

Con respecto a la alta capacidad resolutiva de la CPRE, de acuerdo con publicaciones recientes el éxito del procedimiento se encuentra entre un 74.4 y un $100 \%$. Esto quiere decir que nuestro centro hospitalario se encuentra dentro de los estándares de calidad para la realización de $\mathrm{CPRE}^{8}$.
Cuando analizamos las complicaciones, Magalhaes (2015) reporta dentro de sus complicaciones posteriores a la realización de la CPRE pancreatitis entre un 1.3 y un $6.7 \%$, sangrado entre un 0.3 y un $2 \%$ y perforación entre un 0.1 y un $1.1 \%$. Si se compara con nuestros resultados obtenidos, se encuentran dentro de los rangos permisivos, esto refleja que la morbilidad al momento del estudio no se ve incrementada a pesar de la realización de procedimientos en tiempos de contingencia ${ }^{9}$.

Se presentó en nuestro estudio una canulación difícil en 7 de cada 10 procedimientos, definiéndolo como aquel con incapacidad de canular el conducto biliar a los 5 minutos o que presente canulación u opacificación del conducto pancrático. Castro (2017) menciona que la canulación selectiva es un paso crucial para determinar el éxito de una CPRE, evidenciando que hasta un $20 \%$ de la canulación no se logrará mediante técnicas convencionales, ameritando técnicas avanzadas para lograr el acceso biliar o pancreático ante el fracaso de la técnica convencional. Esto arroja que el uso de las técnicas utilizadas en nuestra unidad de endoscopia han sido las necesarias para disminuir el porcentaje de canulación fallida ${ }^{10}$.

El riesgo potencial de infección por SARS-CoV-2 en pacientes de endoscopia en un entorno de emergencia se debe valorar de acuerdo con los síntomas (p. ej., tos, fiebre, disnea o diarrea), la presencia o no de contacto con alguien positivo y en aquellos con permanencia en áreas de alto riesgo durante los 14 días anteriores. En nuestro estudio, de los pacientes sometidos a CPRE solo dos presentaron síntomas compatibles con COVID-19, clasificándolos como de riesgo intermedio (con síntomas leves o sin presencia de síntomas, pero con antecedente de contacto con alguien SARS-CoV-2 positivo), en los cuales se realizaron pruebas de PCR-RT para la confirmación, siendo negativas en ambos pacientes.

Durante la realización de todos los estudios el personal médico y de enfermería utilizó las medidas y los EPP recomendados y disponibles, además de la limpieza y reprocesamiento del equipo de endoscopia y descontaminación de salas posterior a cada estudio. Esto ha permitido hasta el momento que todo el personal se mantenga asintomático.

\section{Conclusiones}

La realización de la CPRE es factible y segura, sin incremento de complicaciones durante la época de COVID-19, y aunque el apego a las medidas de 
seguridad y el estado clínico del paciente fueron los principales retos durante la contingencia, no afectó las tasas de éxito endoscópico, logrando resultados satisfactorios, con riesgo nulo de contagio para el personal de la salud.

\section{Financiamiento}

La presente investigación no ha recibido ayudas específicas provenientes de agencias del sector público, sector comercial o entidades sin ánimo de lucro.

\section{Conflicto de intereses}

Los autores declaran no tener conflicto de intereses alguno.

\section{Responsabilidades éticas}

Protección de personas y animales. Los autores declaran que para esta investigación no se han realizado experimentos en seres humanos ni en animales.

Confidencialidad de los datos. Los autores declaran que en este artículo no aparecen datos de pacientes.
Derecho a la privacidad y consentimiento informado. Los autores declaran que en este artículo no aparecen datos de pacientes.

\section{Bibliografía}

1. Otero W, Gómez M, Ángel LA, Ruiz Ó, Marulanda H, Riveros J, et al. Basic considerations regarding endoscopic procedures during the COVID-19 pandemic. Rev Colomb Gastroenterol. 2020;35(1) 65-75.

2. Perisetti A, Garg S, Inamdar S, Tharian B. Role of face mask in preventing bacterial exposure to the endoscopist's face. Gastrointest Endosc. 2019;90(5):859.

3. Brandabur J, Leggett J, Wang L, Bartles R, Baxter L, Diaz G, et al. Surveillance of guideline practices for duodenoscope and linear echoendoscope reprocessing in a large healthcare system. Gastrointest Endosc. 2016;84:392-9.

4. Bove V, Schepis T, Boskoski I, Landi R, Orlandini B, Pontecorvi V, et al. Bilio-pancreatic endoscopy during COVID-19 pandemic. Ther Adv Gastroenterol. 2020;13(1):1-11.

5. Gómez-Zuleta M, Melgar C, Arbeláez V. ¿Es la edad un factor que incide en las complicaciones por CPRE? Rev Col Gastroenterol. 2010;25(4):349-53.

6. Buxbaum J, Abbas S, Sultan S, Fishman D, Qumseya B, Cortessis V, et al. ASGE guideline on the role of Endoscopy in the Evaluation management of choledocholithiasis. Gastrointest Endosc. 2019;89(6):1075-105.

7. Ripari G, Wulfson A, Guerrina C, Perroud H. Correlación entre predictores de litiasis coledociana y los hallazgos en la colangiopancreatografía retrógrada endoscópica. De las guías a la práctica. Acta Gastroenterol Latinoam. 2017;47(4):269-76.

8. De Jesús-Flores A, Guerrero-Martínez GA. Impacto del protocolo propuesto por la American Society for Gastrointestinal Endoscopy en pacientes de alto riesgo de coledocolitiasis en el Hospital Regional ISSSTE Puebla en México. Cir Cir. 2019;87:423-27.

9. Magalhaes J, Rosa B, Cotter J. Endoscopic retrograde cholangiopancreatography for suspected choledocholithiasis: from guidelines to clinical practice. World J Gastrointest Endosc. 2015;16:128-4.

10. Castro-Gómez J, Téllez-Ávila F. Advances techniques in difficult biliary cannulation. Endoscopia. 2017;29(1):39-46. 